\title{
Global Solutions and Quenching to a Class of Quasilinear Parabolic Equations ${ }^{1}$
}

\author{
Bei Hu and Hong-Ming Yin \\ Department of Mathematics, \\ University of Notre Dame, Notre Dame, IN 46556
}

\begin{abstract}
In this paper we consider a quasilinear parabolic equation $a\left(u_{x}\right) u_{t}=u_{x x}\left(a(s) \geq a_{0}>0\right)$ subject to appropriate initial and boundary conditions. This equation can be used to describe the uni-directional motion of fluid in soft tissue. A criterion is found to ensure the global solvability or finite time quenching (i.e. $u_{x}$ becomes unbounded in finite time). A concrete example is that for $a(s)=(1+s)^{p}$, the problem has a global solution if $p \leq 2$ while the finite time quenching occurs if $p>2$. The profile near the quenching time such as the single point quenching and the quenching rate are also studied. Partial results are generalized into several space dimensions.
\end{abstract}

\footnotetext{
${ }^{1}$ This work is partially supported by US National Science Foundation Grant DMS 90-24986 and NSERC of Canada.
} 


\section{Introduction}

In the study of motion of fluid in soft tissue such as articular cartilage, the deformation of the solid phase plays a significant role. Recently, Holmes [3, 8] et. al based on the mixture theory derived a model equation which can describe the uni-directional motion of fluid in soft tissue. The reader can consult [7] for more physical background of the model. After normalizing physical constants, the equation can be expressed as follows:

$$
a\left(u_{x}\right) u_{t}=u_{x x}
$$

where $u(x, t)$ represents the displacement of the soft tissue. Obviously, if one writes the equation (1.1) in a traditional way

$$
u_{t}=\frac{1}{a\left(u_{x}\right)} u_{x x}
$$

one can see that the equation becomes degenerate if $a(s) \rightarrow \infty$ as $s \rightarrow \infty$.

In the present paper we shall study the equation $(1.1)$ in $Q_{T}=(0,1) \times(0, T]$ subject to

$$
\begin{aligned}
& u(0, t)=0, \quad 0<t<T ; \\
& u(1, t)=g(t), \quad 0<t<T ; \\
& u(x, 0)=0, \quad x \in(0,1),
\end{aligned}
$$

where $g(t)$ is non-negative and monotone increasing.

It will be seen that the global existence or finite time quenching (i.e. $u_{x}$ becomes unbounded) depends on two quantities: $G^{*}$ and $G^{* *}$, where

$$
G^{*}=F(0) \text { and } F(s)=\int_{s}^{\infty} \frac{1}{a(\xi)} d \xi
$$

and $G(q)=F^{-1}(q)$, the inverse of the function $F(s)$ while

$$
G^{* *}=\int_{0}^{G^{*}} G(q) d q .
$$

A completed description is obtained to ensure the global solvability or the finite time quenching. As a concrete example of our results, we take $a(s)=(1+s)^{p}$. Then the results show that the problem (1.1)-(1.4) has a unique solution globally in time if $p \leq 2$, and the quenching occurs in finite time if $p>2$ and $\sup _{t>0}[g(t)-M(t)]>0$, where $M(t)$ is a function depending only on the known data (see the definition of $M(t)$ below).

Assuming $g(t)$ is smooth, we have the following chart

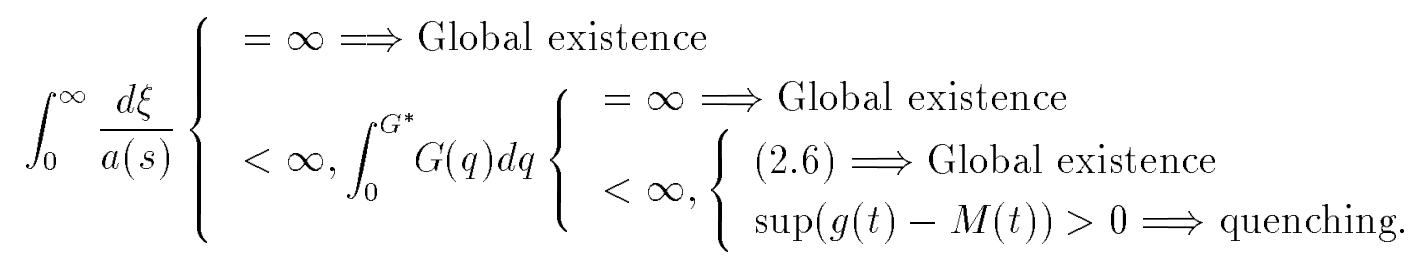


A special case, where $a(s)=e^{s}$, was studied by J. Bell et al [1], which is also the original motivation of the present work. An interesting feature for the equation (1.1) is that there is no source appearing in the equation and the quenching is caused solely by the nonlinearity in the leading coefficient of the equation. On the other hand, if we introduce a new variable $v(x, t)=u_{x}(x, t)$, then $v$ satisfies

$$
\begin{aligned}
& a(v) v_{t}=v_{x x}-\frac{a^{\prime}(v)}{a(v)} v_{x}^{2} \quad \text { in } Q_{T}, \\
& v_{x}(0, t)=0 \text { for } 0 \leq t \leq T, \\
& v_{x}(1, t)=g^{\prime}(t) a(v) \quad \text { for } 0 \leq t \leq T, \\
& v(x, 0)=0 \text { for } 0 \leq x \leq 1 .
\end{aligned}
$$

Since $g^{\prime}(t) \geq 0$, there is indeed a nonlinear heat supply through the boundary (cf. [7]) if one regards $v(x, t)$ as temperature. The argument used in this paper is based on the maximum principle. Various auxiliary functions are constructed as lower or upper solutions of Equation (1.1). A generalization to higher space dimension is also discussed in the present work.

The paper will be organized in the following way. Section 2 deals with global solvability. Section 3 is concerned with finite time quenching. In Section 4, the profile near the quenching time will be studied. Various generalizations will be given in Section 5 .

Except for Section 5, we shall make the following assumptions.

(H1). $a \in C^{1}(-\infty, \infty)$ and $a(s) \geq a_{0}>0$ for all $s$.

(H2). $g \in C^{1}[0, \infty), g(0)=0$ and $g^{\prime}(t) \geq 0$ for $t>0$.

\section{Global Solvability}

We define the function $F(s)$ as in Section 1. Consider the first case

$$
G^{*}=F(0)=\int_{0}^{\infty} \frac{1}{a(\xi)} d \xi=\infty .
$$

Theorem 2.1: Under the assumptions (H1), (H2) and (2.1), the system (1.1)-(1.4) has a unique classical solution $u(x, t)$ for $0 \leq t<\infty$.

Next, we consider the case

$$
G^{*}=F(0)=\int_{0}^{\infty} \frac{1}{a(\xi)} d \xi<\infty .
$$

In this case, $F(s)$ is well defined on $[0, \infty)$. Since $F^{\prime}(s)<0$ for all $s$, and $F(0)=G^{*}$ and $F(\infty)=0$, the inverse function of $F$ is well defined on $\left(0, G^{*}\right]$, and we set

$$
G(q)=F^{-1}(q), q \in\left(0, G^{*}\right] .
$$


Theorem 2.2: Let the assumptions (H1) (H2) and (2.2) be in force. If

$$
G^{* *}=\int_{0}^{G^{*}} G(q) d q=\infty
$$

then the system (1.1)-(1.4) has a unique classical solution $u(x, t)$ for $0 \leq t<\infty$.

One will see in Section 3 that in the case of (2.2), quenching may occur if we drop the assumption (2.3).

The existence of a unique classical solution for small time is a standard proof for such a parabolic equation (cf. [5] for example). Therefore, the global existence will follow if an a priori estimate in $C^{2+\alpha, 1+\frac{\alpha}{2}}\left(\bar{Q}_{T}\right)$ is available. It is clear that the only estimate needed for this system is the $L^{\infty}$-estimate of $u_{x}$. Once this estimate is established, one can easily get estimates for higher order derivatives by the theory for parabolic equations.

Proof of Theorem 2.1: We take $T>0$. By the maximum principle, we immediate conclude

$$
\begin{aligned}
& 0 \leq u(x, t) \leq g(T) \text { for } 0 \leq x \leq 1,0 \leq t \leq T \\
& 0 \leq u_{t}(x, t) \leq\left\|g^{\prime}\right\|_{C[0, T]}
\end{aligned}
$$

For each $t \in[0, T]$, there exists $x_{1} \in[0,1]$ such that

$$
u_{x}\left(x_{1}, t\right)=\int_{0}^{1} u_{x}(\xi, t) d \xi=g(t) .
$$

¿From (H1) and the equation (1.1),

$$
0 \leq \frac{u_{x x}}{a\left(u_{x}\right)} \leq\left\|g^{\prime}\right\|_{C[0, T]}
$$

Therefore by integrating over interval $\left(x, x_{1}\right)$ or $\left(x_{1}, x\right)$, we have

$$
\left|\int_{g(t)}^{u_{x}(x, t)} \frac{d \xi}{a(\xi)}\right|=\left|\int_{u_{x}\left(x_{1}, t\right)}^{u_{x}(x, t)} \frac{d \xi}{a(\xi)}\right| \leq C\left|x-x_{1}\right| \leq C \quad \text { for } 0 \leq x \leq 1,0<t<T .
$$

This gives the uniform bound for $u_{x}(x, t)$ if the condition (2.1) holds. This proves Theorem 2.1.

Proof of Theorem 2.2: To estimate $\left\|u_{x}\right\|_{L^{\infty}\left(Q_{T}\right)}$, we only need to obtain an upper bound for $u_{x}(1, t)$ since $u_{x}(x, t) \geq 0$ and $u_{x x}(x, t) \geq 0$. The idea is to construct a subsolution $v(x, t)$ of (1.1)-(1.4) with $v(1, t)=g(t)$. Let $A=\left\|g^{\prime}\right\|_{C[0, T]}$ and consider the function

$$
h(x)=\int_{0}^{1-x} G(A \xi+F(B)) d \xi .
$$

By the definition of $F(s), \lim _{B \rightarrow \infty} F(B)=0$. We take $B$ to be large enough such that $F(B)<G^{*} / 2$. Then $h(x)$ is well defined for $1-\delta \leq x \leq 1$, where $\delta=\min \left[1, G^{*} /(2 A)\right]$. 
Notice that the condition (2.3) implies that $\lim _{B \rightarrow \infty} h(1-\delta)=\infty$. Therefore $h(1-\delta)>g(T)$, if we take $B$ to be large enough.

We now let

$$
v(x, t)=g(t)-h(x) \text { for } 1-\delta<x<1,0<t<T .
$$

On the parabolic boundary, $v(1-\delta, t) \leq 0 \leq u(1-\delta, t), v(x, 0) \leq 0=u(x, 0)$ and $v(1, t)=$ $g(t)=u(1, t)$. In the interior, $v$ satisfies

$$
\begin{aligned}
& a\left(v_{x}\right) v_{t}-v_{x x} \\
& =a\left(-h^{\prime}(x)\right) g^{\prime}(t)+h^{\prime \prime}(x) \\
& \leq a\left(-h^{\prime}(x)\right)\left(A+\frac{h^{\prime \prime}(x)}{a\left(-h^{\prime}(x)\right)}\right) \\
& =a\left(-h^{\prime}(x)\right)\left[A-h^{\prime \prime}(x) F^{\prime}\left(-h^{\prime}(x)\right)\right] \\
& =A a\left(-h^{\prime}(x)\right)\left\{1-G^{\prime}[A(1-x)+F(B)] F^{\prime}[G(A(1-x)+F(B))]\right\} \\
& =0 .
\end{aligned}
$$

It follows by the comparison principle that $v(x, t) \leq u(x, t)$ and hence $u_{x}(1, t) \leq v_{x}(1, t)=$ $G(F(B))=B$ for $0<t<T$. Thus by the maximum principle $0 \leq u_{x}(x, t) \leq B$ for all $0 \leq x \leq 1,0 \leq t \leq T$. Since $T$ is arbitrary, Theorem 2.2 follows.

When the condition (2.3) fails, the problem (1.1)-(1.4) does not have a global solution in general (See Theorem 3.1 in Section 3). However, we have some sufficient conditions for the global existence in the case where the condition (2.3) on $g(t)$ does not hold.

Theorem 2.3: Let the assumptions (H1), (H2) and (2.2) be in force and $G^{* *}<\infty$. If

$$
g^{\prime \prime}(t) \leq 0, \quad g(t) g^{\prime}(t)<\int_{0}^{g^{\prime}(t)} G(\xi) d \xi, \quad g^{\prime}(t)<G^{*} \quad \text { for } 0<t<\infty
$$

then the system (1.1)-(1.4) has a unique classical solution $u(x, t)$ for $0 \leq t<\infty$.

Proof: The proof is similar to that of Theorem 2.2. Let $T>0$ be fixed and consider the function

$$
h(x, t ; B)=\int_{0}^{1-x} G\left(g^{\prime}(t) \xi+F(B)\right) d \xi \text { for } 0 \leq x \leq 1,0 \leq t \leq T .
$$

By assumption (2.6), there exists $\varepsilon>0$ such that

$$
g^{\prime}(t) \leq G^{*}-\varepsilon \quad \text { for } 0 \leq t \leq T
$$

It follows that $h(0, t ; B)$ is well defined if $B$ is large enough. By assumption (2.6),

$$
h(0, t ; \infty)=\int_{0}^{1} G\left(g^{\prime}(t) \xi\right) d \xi=\frac{1}{g^{\prime}(t)} \int_{0}^{g^{\prime}(t)} G(\xi) d \xi>g(t) \quad \text { for } 0 \leq t \leq T ;
$$

this implies that

$$
h(0, t) \equiv h(0, t ; B)=\int_{0}^{1} G\left[g^{\prime}(t) \xi+F(B)\right] d \xi>g(t) \quad \text { for } 0 \leq t \leq T,
$$


provided $B$ is sufficiently large. Since $g^{\prime \prime}(t) \leq 0$ and $G^{\prime}(q)<0$, we have

$$
h_{t}(x, t) \geq 0 .
$$

We now let

$$
v(x, t)=g(t)-h(x, t) \text { for } 0<x<1,0<t<T .
$$

Then it is easy to verify that $v(x, t) \leq u(x, t)$ on the parabolic boundary of $(0,1) \times(0, T)$. In the interior, $v$ satisfies

$$
\begin{aligned}
a\left(v_{x}\right) & v_{t}-v_{x x} \\
& =a\left(-h_{x}(x, t)\right) g^{\prime}(t)+h_{x x}(x, t)-a\left(-h_{x}(x, t)\right) h_{t}(x, t) \\
& \leq a\left(-h_{x}(x, t)\right) g^{\prime}(t)+\frac{h_{x x}(x, t)}{a\left(-h_{x}(x, t)\right)} \\
& =a\left(-h_{x}(x, t)\right)\left[g^{\prime}(t)-h_{x x}(x, t) F^{\prime}\left(-h_{x}(x, t)\right)\right] \\
& =g^{\prime}(t) a\left(-h_{x}(x, t)\right)\left\{1-G^{\prime}\left[g^{\prime}(t)(1-x)+F(B)\right] F^{\prime}\left[G\left(g^{\prime}(t)(1-x)+F(B)\right)\right]\right\} \\
& =0 .
\end{aligned}
$$

It follows by the comparison principle that $v(x, t) \leq u(x, t)$ and hence $u_{x}(1, t) \leq v_{x}(1, t)=$ $G(F(B))=B$ for $0<x<T$. Thus the maximum principle implies that $0 \leq u_{x}(x, t) \leq B$ for all $0 \leq x \leq 1,0 \leq t \leq T$. Theorem 2.3 now follows since $T$ is arbitrary.

The condition (2.6) is optimal on the order of magnitude as $t \rightarrow \infty$. In fact, we have Theorem 2.4: Assume that the conditions (H1) , (2.2) hold and $G^{* *}<\infty$, then there exists a function $g(t)$ satisfying (2.6) such that

$$
\limsup _{t \rightarrow \infty} \frac{M(t)}{g(t)} \leq 1+\beta,
$$

where $0 \leq \beta \leq 1$ and

$$
M(t) \equiv \inf _{0<A<G^{*}}\left(A t+\frac{1}{A} \int_{0}^{A} G(\xi) d \xi\right) .
$$

Moreover, we can take $\beta=0$ if $a(s)=e^{s}, \beta=1 / p$ if $a(s)=(1+s)^{p}$.

Remark: It will be shown in Section 3 that if $g(t)>M(t)$ for some $t=t_{0}$, then quenching will occur at a finite time.

Proof: Take $\varepsilon_{0}>0$ to be small enough. Then there exists $T>0$ such that

$$
\varepsilon_{0} T=\left(1-\varepsilon_{0}\right) \frac{1}{\varepsilon_{0}} \int_{0}^{\varepsilon_{0}} G(\xi) d \xi=\left(1-\varepsilon_{0}\right) \int_{0}^{1} G\left(\varepsilon_{0} \xi\right) d \xi
$$

We now define $g(t)=\varepsilon_{0} t$ for $0 \leq t \leq T$ and define $g(t)(t \geq T)$ as the solution of the following ordinary differential equation (ODE).

$$
\begin{aligned}
& g(t)=\left(1-g^{\prime}(t)\right) \int_{0}^{1} G\left(g^{\prime}(t) \xi\right) d \xi \equiv \varphi\left(g^{\prime}(t)\right), \\
& g(T)=\varepsilon_{0} T .
\end{aligned}
$$


Clearly, $\varphi(s)$ is defined for $0<s \leq \varepsilon_{0}\left(<G^{*}\right), \varphi(0)=\infty($ since $G(0)=\infty)$ and $\varphi^{\prime}(s)<0$. Therefore $g^{\prime}(T+0)=\varepsilon_{0}, g^{\prime}(t)>0$ for all $t>0$ and $g^{\prime}$ is continuous. Differentiating the equation (2.8), we immediately obtain $g^{\prime \prime}(t)<0$ and thus the condition $(2.6)$ is satisfied.

To show (2.7), we take $A=g^{\prime}(t)$ in the definition for $M(t)$ and obtain

$$
\frac{M(t)}{g(t)} \leq \frac{t g^{\prime}(t)}{g(t)}+\frac{1}{g(t)} \int_{0}^{1} G\left(g^{\prime}(t) \xi\right) d \xi=\frac{t g^{\prime}(t)}{g(t)}+\frac{1}{1-g^{\prime}(t)}
$$

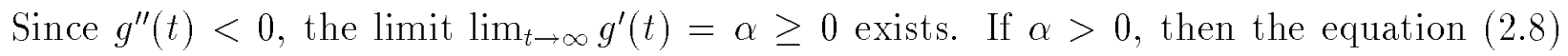
implies that $\lim _{t \rightarrow \infty} g(t)=\varphi(\alpha)$, which is a contradiction to the fact $g(t) \sim \alpha t$ as $t \rightarrow \infty$. Hence $\alpha=0$, i.e.,

$$
\lim _{t \rightarrow \infty} g^{\prime}(t)=0
$$

This equality, together with the equation (2.8), implies that

$$
\lim _{t \rightarrow \infty} g(t)=\varphi(0)=+\infty
$$

We now set $\beta=\limsup _{t \rightarrow \infty} \frac{t g^{\prime}(t)}{g(t)}$. It remains to show that $\beta \leq 1$. Let $\psi$ be the inverse function of $\varphi$. Then $\psi(s)$ is well defined for $\varepsilon_{0} T=\varphi\left(\varepsilon_{0}\right) \leq s<\infty$ and $\psi^{\prime}(s)<0$. It follows that

$$
\int_{\varepsilon_{0} T}^{g} \frac{d \eta}{\psi(\eta)}=t-T
$$

and hence

$$
\begin{aligned}
\beta & =\limsup _{t \rightarrow \infty} \frac{g^{\prime}(t)}{g(t)}\left(T+\int_{\varepsilon_{0} T}^{g} \frac{d \eta}{\psi(\eta)}\right) \\
& =\limsup _{g \rightarrow \infty} \frac{\psi(g)}{g-\varepsilon_{0} T} \int_{\varepsilon_{0} T}^{g} \frac{d \eta}{\psi(\eta)} \leq 1 .
\end{aligned}
$$

In the case $a(s)=e^{s}$, a direct calculation shows that $\beta=0$.

\section{Finite Time Quenching}

Theorem 3.1: Let the assumptions (H1) (H2) and the condition (2.3) be in force. If

$$
\int_{0}^{G^{*}} G(q) d q<\infty
$$

then

$$
g(t) \leq M(t) \equiv \inf _{0<A<G^{*}}\left(A t+\frac{1}{A} \int_{0}^{A} G(\xi) d \xi\right)
$$

is a necessary condition for the existence of a classical solution.

An immediate consequence is

Corollary 3.2: Under the same conditions as Theorem 3.1, the quenching occurs in finite 
time if $g\left(t_{0}\right)>M\left(t_{0}\right)$ for some $t_{0}>0$, and the quenching time $T<t_{0}$.

Proof of Theorem 3.1: Let $0<A<G^{*}$ and consider the function

$$
h(x)=\int_{0}^{1-x} G(A \xi) d \xi \quad \text { for } 0 \leq x \leq 1,
$$

By the assumption (2.2), the function $h(x)$ is well defined. Set

$$
v(x, t)=A t-h(x)+h(0), \quad \text { for } 0 \leq x \leq 1,0 \leq t \leq T .
$$

Then

$$
\begin{aligned}
v_{t}(x, t) & =A \\
v_{x}(x, t) & =-h^{\prime}(x)=G[A(1-x)] \geq 0 \\
v_{x x}(x, t) & =-h^{\prime \prime}(x)=-A G^{\prime}[A(1-x)] \\
= & -\frac{A}{F^{\prime}(G(q))}=A a(G(q))=A a\left(-h^{\prime}(x)\right) .
\end{aligned}
$$

It follows that on $Q_{T}$,

$$
a\left(v_{x}\right) v_{t}-v_{x x}=A a\left(-h^{\prime}(x)\right)\left(1+\frac{h^{\prime \prime}(x)}{A a\left(-h^{\prime}(x)\right)}\right)=0 .
$$

On the parabolic boundaries $v(0, t)=A t \geq u(0, t)$ and as $h^{\prime}(x) \leq 0, v(x, 0)=h(0)-h(x) \geq$ $0=u(x, 0)$. Moreover, since $F(\infty)=0$, we have $G(0)=\infty$ and so $v_{x}(1, t)=G(0)=$ $+\infty>u_{x}(1, t)$ if the problem (1.1)-(1.4) has a solution $u(x, t)$ on $[0, T]$. Now it follows from comparison principle that

$$
u(x, t) \leq v(x, t) \text { for } 0 \leq x \leq 1,0 \leq t \leq T
$$

Especially, we have

$$
g(t)=u(1, t) \leq v(1, t)=A t+h(0)=A t+\int_{0}^{1} G(A \xi) d \xi=A t+\frac{1}{A} \int_{0}^{A} G(\xi) d \xi .
$$

Minimizing over $A$ yields the result of the theorem.

As for $a(s)=(1+s)^{p}$, we have

Corollary 3.3: (1) If $p \leq 2$, the problem (1.1)-(1.4) has a unique global solution for any smooth $g(t)$.

(2) If $p>2$, the quenching occurs in finite time if

$$
g(t)>\inf _{0<A<\frac{1}{p-1}}\left\{A t-1+\frac{1}{p-2}(p-1)^{(p-2) /(p-1)} A^{-1 /(p-1)}\right\},
$$

for some $t=t_{0}>0$. The right-hand side of the above inequality is equal to $c t^{1 / p}-1$ for large $t$, and $c$ is a constant $\left(c=p(p-2)^{-(p-1) / p}(p-1)^{-1 / p}\right)$. 


\section{Profile near the Quenching Time}

Let $T$ be the quenching time, that is

$$
T=\sup \left\{t: u_{x}(x, t)<\infty, \text { for all } x \in[0,1]\right\} \text {. }
$$

A point $\left(x_{0}, T\right)$ is said a quenching point if there exists a sequence $\left(x_{n}, t_{n}\right)$ with $t_{n}<T$ such that $\left(x_{n}, t_{n}\right) \rightarrow\left(x_{0}, T\right)$ and $u_{x}\left(x_{n}, t_{n}\right) \rightarrow \infty$ as $n \rightarrow \infty$. Our first result is about the set of quenching points.

Theorem 4.1: The quenching occurs only at $x=1$. Moreover,

$$
\lim _{t \rightarrow T-} u_{x}(1, t)=\infty
$$

Proof: Since $u_{x x}(x, t) \geq 0$, we immediately conclude that

$$
u_{x}(x, t)(1-x) \leq \int_{x}^{1} u_{x}(\xi, t) d \xi=g(t)-u(x, t) .
$$

It follows that $u_{x}(x, t) \leq C /(1-x)$ and therefore quenching occurs only at $x=1$.

If the the second conclusion is not true, then there exists a sequence $t_{n} \uparrow T$ such that $0 \leq u_{x}\left(1, t_{n}\right) \leq C$, where the constant $C$ is independent of $n$. Since $u_{x x} \geq 0$, we obtain $0 \leq u_{x}\left(x, t_{n}\right) \leq C$. It now follows from the equation (1.1) and the estimate (2.5) that

$$
0 \leq u_{x}\left(x, t_{n}\right) \leq C^{*}, 0 \leq u_{x x}\left(x, t_{n}\right) \leq C^{*} \text { for } 0<x<1
$$

where the constant $C^{*}$ is independent of $n$. It follows by local existence theorem that there is a unique classical solution $v$ for $t_{n} \leq t<t_{n}+\delta$, which satisfies the equation (1.1) with the boundary conditions (1.2) (1.3) and initial value taken on $t=t_{n}$ as $v\left(x, t_{n}\right)=u\left(x, t_{n}\right)$. Of course, $v_{x}(x, t)$ is uniformly bounded. The constant $\delta$ depends only on $C^{*}$ and therefore is independent of $n$. It is also clear that $u(x, t) \equiv v(x, t)$ for $t<T$. Since $t_{n} \uparrow T, t_{n}+\delta>T$, provided $n$ is large enough. This is a contradiction since $u_{x}(x, t)$ is unbounded on $[0, T)$.

The next theorem gives the lower bound for the quenching rate.

Theorem 4.2: Suppose that $T$ is the quenching time. Then

$$
\liminf _{t \rightarrow T} u_{x}(x, t) \geq G\left[\left\|g^{\prime}\right\|_{C[0, T]}(1-x)\right] .
$$

Proof: Since quenching occurs, both $F(s)$ and $G(q)$ are well defined. Integrating the equation $-u_{t}=\frac{\partial}{\partial x} F\left(u_{x}\right)$ over $(x, 1)$, we get

$$
\begin{aligned}
F\left(u_{x}(x, t)\right) & =F\left(u_{x}(1, t)\right)+\int_{x}^{1} u_{t}(\xi, t) d \xi \\
& \leq F\left(u_{x}(1, t)\right)+\left\|g^{\prime}\right\|_{C[0, T]}(1-x) .
\end{aligned}
$$


It follows that

$$
u_{x}(x, t) \geq G\left[F\left(u_{x}(1, t)\right)+\left\|g^{\prime}\right\|_{C[0, T]}(1-x)\right] .
$$

Letting $t \rightarrow T$, and observing that $F(\infty)=0$, we conclude the theorem.

In the case $a(s)=(1+s)^{p}$ with $p>2$, Theorem 4.2 implies that

$$
\liminf _{t \rightarrow T} u_{x}(x, t) \geq c(1-x)^{-\frac{1}{p-1}}
$$

for some $c>0$.

We also have the upper bound if we further assume that

$$
g^{\prime}(t)>0 \text { for } t>0
$$

Theorem 4.3: Let (4.1) hold. If $T$ is the quenching time, then

$$
u_{x}(x, t) \leq G\left[c_{0}(1-x)\right] \text { for } \frac{1}{2} \leq x \leq 1, \frac{T}{2}<t<T
$$

for some $c_{0}>0$.

Proof: By assumption (4.1), there exists a constant $c_{0}$ such that $g^{\prime}(t) \geq c_{0}>0$ for $T / 2 \leq$ $t \leq T$. The strong maximum principle implies that

$$
u_{t}(x, t) \geq c_{0} \text { for }(x, t) \in\left\{x=\frac{1}{2}, \frac{T}{2} \leq t<T\right\} \cup\left\{\frac{1}{2} \leq x \leq 1, t=\frac{T}{2}\right\}
$$

if $c_{0}$ is sufficiently small. It follows by maximum principle that

$$
u_{t}(x, t) \geq c_{0} \text { for } \frac{1}{2} \leq x \leq 1, \frac{T}{2}<t<T .
$$

Following the proof of Theorem 4.2 we obtain

$$
u_{x}(x, t) \leq G\left[F\left(u_{x}(1, t)\right)+c_{0}(1-x)\right] \leq G\left[c_{0}(1-x)\right] \quad \text { for } \frac{1}{2} \leq x \leq 1, \frac{T}{2}<t<T .
$$

When $a(s)=(1+s)^{p}$, Theorem 4.3 implies that

$$
u_{x}(x, t) \leq K(1-x)^{-\frac{1}{p-1}}
$$

for some $K>0$.

\section{Generalization to n-dimensional Case}

Let $\Omega \subset R^{n}$ be a bounded domain with smooth boundary $S=\partial \Omega$ and $Q_{T}=\Omega \times(0, T]$. We shall use the standard convention $u_{x}=\left(u_{x_{1}}, \ldots, u_{x_{n}}\right)$, etc. Consider the following system

$$
\begin{aligned}
& a\left(u_{x}\right) u_{t}=\Delta u, \quad \text { in } Q_{T}, \\
& u(x, t)=g(x, t), \quad(x, t) \in S_{T}=S \times[0, T], \\
& u(x, 0)=u_{0}(x), \quad x \in \Omega .
\end{aligned}
$$


We shall assume that $g(x, t) \in C^{2+\alpha, 1+\frac{\alpha}{2}}\left(S_{T}\right)$ and $u_{0}(x) \in C^{2+\alpha}(\bar{\Omega})$ with the following consistency conditions on $S$ :

$$
g(x, 0)=u_{0}(x), a\left(u_{0 x}\right) g_{t}(x, 0)=\Delta u_{0}(x) .
$$

The function $a(p)$ is defined in $R^{n}$ and differentiable. Moreover, $a(p) \geq a_{0}>0$. We first consider an easy case where

$$
a(p) \leq A_{0}\left[1+|p|^{2}\right],
$$

Theorem 5.1: Under the assumption (5.4), the problem (5.1)-(5.3) has a unique classical solution for any $T<\infty$.

Proof: As in Section 2, an application of the maximum principle yields that $u(x, t)$ and $u_{t}(x, t)$ are a priori bounded on $\bar{Q}_{T}$. For $t \in(0, T]$ fixed, we regard the equation (5.1) as an elliptic equation on $\Omega$ :

$$
\Delta u=u_{t} a\left(u_{x}\right)
$$

subject to a Dirichlet condition (5.2) on $S$. By the results in [2], we have

$$
\left\|u_{x}\right\|_{L^{\infty}\left(Q_{T}\right)} \leq C
$$

where $C$ depends only on known data.

Hence by applying the standard parabolic theory (cf. [5]), we obtain the desired result.

In general, we do not have a similar characterization as that for one space dimension. Here we state a sufficient condition which ensures the finite time quenching.

Theorem 5.2: If there exists a $p_{i}(1 \leq i \leq n)$ such that

$$
a(p) \geq c_{0}\left(1+\left|p_{i}\right|^{2+\delta}\right)
$$

for some $c_{0}>0$ and $\delta>0$, then the quenching occurs in finite time, provided that the initial and boundary data are chosen properly.

Proof: We only sketch the proof. Consider the equations

$$
a\left(u_{x}\right) u_{t}=\Delta u
$$

and

$$
c_{0}\left(1+\left|w_{x_{\imath}}\right|^{2+\delta}\right) w_{t} \geq \Delta w
$$

With the initial data and boundary data of $w$ majorizing that of $u$, the comparison principle implies

$$
u(x, t) \leq w(x, t) \text { on } \overline{Q_{T}},
$$


if $u_{t} \geq 0$. So we choose the initial and boundary data such that $u_{t} \geq 0$. We only need to construct an supersolution for the equation

$$
c_{0}\left(1+\left|w_{x_{i}}\right|^{2+\delta}\right) w_{t}=\Delta w
$$

Without loss of generality, we assume the following

$$
\begin{gathered}
p_{i}=p_{1} \\
x_{1}<1 \quad \text { for all }\left(x_{1}, x_{2}, \cdots, x_{n}\right) \in \Omega, \\
(1,0, \cdots, 0) \in \partial \Omega .
\end{gathered}
$$

Take $\eta>0$ to be small enough, and set

$$
S_{1}=\partial \Omega \cap\left\{x_{1} \leq 1-\eta\right\}, \quad S_{2}=\partial \Omega \cap\left\{1-\eta \leq x_{1} \leq 1\right\}
$$

We set $g(x, t)=0$ on $S_{1} \times[0, T]$. Suppose that $u(x, t)$ is a classical solution, then we can compare it with a one-dimensional supersolution, as in the proof of Theorem 3.1. It follows that

$$
u(x, t) \leq M(t) \text { on } Q_{T} \cap\left\{x_{1} \leq 1-\eta\right\},
$$

where $M(t)$ is independent of $T$ and the data on $S_{2} \times[0, T]$. Since $\partial \Omega$ is $C^{2+\alpha}, S_{2}$ has a representation

$$
x_{1}=h\left(x_{2}, \cdots, x_{n}\right)
$$

provided $\eta$ is small enough. We now consider the auxiliary function

$$
v(x, t)=\varphi\left(x_{1}-h\left(x_{2}, \cdots, x_{n}\right), t\right)+\sup _{0 \leq t \leq T} M(t) \quad \text { in } Q_{T} \cap\left\{x_{1}>1-\eta\right\} .
$$

Notice that $h_{x_{i}}(0,0, \cdots, 0)=0(i=2, \cdots, n),|\nabla h| \leq 1 / 2$ if $\eta$ is small enough. We choose $\varphi(y, t)$ to be of the form

$$
\varphi(y, t)=B t+b(y) \quad \text { for } y_{0} \equiv 1-\eta-\max _{S_{2}} h\left(x_{2}, \cdots, x_{n}\right)<y<0
$$

where $B$ and $b(y)$ satisfy

$$
\begin{aligned}
& \frac{B}{2} \geq \sup _{p_{1}}\left(\frac{\left|p_{1}\right|}{c_{0}\left(1+\left|p_{1}\right|^{2+\delta}\right)}\right)\left|\sum_{1=2}^{n} h_{x_{i} x_{i}}\right|, \\
& \frac{B}{2}=\frac{5}{4 c_{0}} \frac{b^{\prime \prime}(y)}{1+\left(b^{\prime}(y)\right)^{2+\delta}} \\
& b\left(y_{0}\right)=0 \\
& b^{\prime}(0)=\infty .
\end{aligned}
$$


¿From the same proof of Theorem 3.1, we know that such a function $b(y)$ exists, and $b^{\prime}(y)>$ $0, b^{\prime \prime}(y)>0$ if $\eta$ is small enough. With the above choice of $\varphi$, we see that $v(x, t)$ is a supersolution, and hence

$$
u(x, t) \leq v(x, t) \quad \text { on } S_{2} \times[0, T] .
$$

Thus the solution quenches in finite time if the data on $S_{2} \times[0, T]$ violates $(5.5)$.

\section{References}

1. J. Bell, A. Friedman and A.A. Lacey, On solutions to a quasilinear diffusion problem from the study of soft tissue, SIAM J. on Applied Math., 51 (1991), 484-493.

2. D. Gilbarg and N.S. Trudinger, Elliptic Partial Differential Equations of Second Order, Springer-Verlag, Berlin, 1983.

3. H. M. Holmes, Comparison theorems and similarity solution approximations for a nonlinear diffusion equation arising in the study of soft tissue, SIAM on applied Math., 44 (1984), 545-556.

4. H. M. Holmes, A nonlinear diffusion equation arising in the study of soft tissue, Quart. J. Appl. Math., 61 (1983), 209-220.

5. O.A. Ladyzenskaya, V. Solonnikov and N. Uralceva, Linear and Quasilinear equations of parabolic type, AMS translation math. Monographys, vol. 23, Providence, R.I., 1968 .

6. H. M. Yin, Blowup versus global existence for a class of nonlinear parabolic equations, Nonlinear Analysis, TMA, submitted.

7. W. Walter, On existence and nonexistence in the large of solutions of parabolic differential equations with a nonlinear boundary conditions, SIAM J. on Math. Anal., 6 (1974), $85-90$.

8. V. Mow, H.M. Holmes and W. M. Lai, Fluid transport and mechanical properties of articular cartilage: a review, J. Biomech., 17 (1984), 377-394. 